\title{
50 AÑOS DE LA PÍLDORA ANTICONCEPTIVA
}

El nacimiento de la píldora anticonceptiva se remonta a 50 años atrás, y pocos acontecimientos en la historia de la investigación farmacológica y el desarrollo de medicamentos han tenido efectos tan profundos sobre aspectos tan variados y sensibles del diario vivir como son la sexualidad y la reproducción. También ha influido en la opinión explicitada de diferentes grupos de pensamiento y decisión respecto a su utilización, entre ellos las comunidades científica y religiosa, agrupaciones políticas, otros ideólogos y aún más importante, por el común de los ciudadanos, especialmente las mujeres.

Remedando una frase famosa de esa década, se podría decir que quizás nunca en la historia de la humanidad, una cosa tan pequeña ha tenido consecuencias tan grandes. El 23 de abril de 1960 la "píldora" recibía el permiso para ser dispensada y vendida explícitamente como anticonceptivo oral en los Estados Unidos de Norteamérica. Empezaba así una "revolución" que incidiría en los años siguientes, y hasta el día de hoy, en la vida de millones de mujeres de todo el mundo, y por ello, necesariamente, en los hombres, en las familias y en las sociedades de todas las naciones.

En este año 2010 , se cumple el $50^{\circ}$ aniversario de ese hecho, y vale la pena hacer un breve recorrido sobre las etapas que llevaron a su aprobación, sobre el significado que la misma ha tenido y sigue teniendo en la vida de millones de personas, y sobre algunas consecuencias más o menos conocidas.

Como en tantos otros descubrimientos, su origen deriva de en un hecho fortuito ocurrido en las selvas tropicales de México en los años treinta del siglo pasado. Allí, el profesor de química Russell Marker, que se encontraba de vacaciones, estaba experimentando con un grupo de esteroides vegetales conocidos como sapogeninas, cuando descubrió un proceso químico que transformaba la sapogenina diosgenina en progesterona $(1,2)$, es decir, en la hormona sexual femenina que tan intensamente se estaba buscando, naciendo así la posibilidad de desarrollar una sustancia sintética con actividades similares a la progesterona que pudiera administrarse por vía oral y que fuera capaz, en las dosis usadas, de interferir con el proceso cíclico de la ovulación y la fecundación.

Toda investigación requiere, desde luego, una serie de inversiones económicas y el apoyo de personas interesadas en conseguir resultados eficaces, y en relación a esto debemos recordar, entre otros, los nombres de Margaret Sanger, quien consiguió financiamiento para continuar las investigaciones de Gregory Pincus, Min Chueh Chang y John Rock (3-5).

Los primeros resultados permitieron preparar una píldora en 1955, que luego recibió el nombre comercial de Enovid. La píldora se basaba en una combinación estro-progestínica, en la que se mezclaban mestranol (150 microgramos) y norethynodrel (10 miligramos), aunque luego las cantidades de ambas sustancias fueron rebajadas. Del laboratorio se pasó en seguida a la fase de experimentación sobre mujeres. Los experimentos se iniciaron en 1956 en Puerto Rico, y el año siguiente en Haití y en Ciudad de México.

En un ambiente de presiones y de expectativas crecientes, la "Food and Drug Administration" (FDA), el organismo de Estados Unidos que da los permisos necesarios para vender y usar productos farmacéuticos, dio en 1957 la luz verde para el uso de Enovid, no como anticonceptivo, sino como fármaco para regular la menstruación. Tres años después, el 23 de junio de 1960, la píldora recibía el permiso para ser vendida explícitamente como anticonceptivo oral. De este modo, en Estados Unidos empezaba una revolución que iba a incidir profundamente en la vida de millones de mujeres de todo el planeta, iniciándose así una nueva época para la humanidad.

Lo novedoso fue que ahora las mujeres podrían obtener la liberación de su dependencia de su vida sexual con respecto a la maternidad. Con el primer medicamento destinado a ser usado en gente sana y además durante períodos prolongados de tiempo, se consigue un gran paso, progreso indiscutible hacia la búsqueda de la igualdad de la mujer con el hombre, liberándola de la amarra difícil de romper 
de sexualidad y embarazo. En otras palabras, la mujer llegaba a conseguir una situación que hasta ahora era sólo privilegio inequitativo del varón, cual era la de ejercer su sexualidad sin el riesgo de la llegada de un hijo, sin quedar embarazada.

Desde entonces, los laboratorios han trabajado intensamente para crear una variedad de versiones más seguras en el campo de los anticonceptivos orales, y con menos efectos colaterales. A corto andar, Hershel Smith en 1963 sintetizó el racemato de norgestrel gonano, siendo la primera síntesis total de un gestágeno. Poco tiempo después se aisló su componente biológicamente activo, el levonorgestrel.

En la década de los setenta fue observado y demostrado que los anticonceptivos orales con sus altas dosis conferían además algunos beneficios para la salud, pero también un aumento en el riesgo cardiovascular. Pronto fue evidenciada una relación directa entre la concentración estrogénica y los eventos cardiovasculares. Fue el British Committee on Safety of Drug quien comunicó que los productos con más estrógenos tenían relación con más informes de embolia pulmonar, trombosis de venas profundas, trombosis cerebral y trombosis coronaria. A su vez el Kingdom's Royal College of General Practitioners, informó que con la reducción del contenido de estrógenos se disminuía la incidencia de trombosis en un $25 \%$, por lo tanto los anticonceptivos orales pasaron de las altas concentraciones estrogénicas a las inferiores a 50 ug por tableta. Dionne y Vickerson en 1974 (6), sugirieron reducir aún más los estrógenos - a 30 ug -, siendo estas denominadas microdosis y anunciaron que a estas dosis el riesgo relativo de trombo-embolismo es similar al de la población no usuaria de anticonceptivos orales. Hoy día están disponibles píldoras que incluyen 20, e incluso 15 ug de etinilestradiol, sustancia que ha seguido siendo el estrógeno más utilizado.

El levonorgestrel fue la base de los primeros anticonceptivos orales combinados que apareciendo por el año 1973 incluían 30 ug de etinilestradiol. Para la misma época se observó que algunos gestágenos, especialmente los 17-a -hidroxi derivados, al combinarlos con estrógenos también predisponían a enfermedad cardiovascular. Para disminuir estos efectos metabólicos adversos se sintetizaron nuevos progestágenos y además se disminuyó considerablemente su concentración.

Durante la década de los ochenta se introdujeron el gestodeno, norgestimato y desogestrel, también del grupo de los gonano, que tienen al parecer un impacto metabólico favorable mucho mayor que sus predecesores. En 1998, son introducidos en
Colombia los anticonceptivos orales combinados de muy baja dosis, que asocian 20 ug de etinilestradiol y 75 ug de gestodeno. Actualmente ya se está utilizando la combinación etinilestradiol con drospirenona, gestágeno con actividad antimineralocorticoide. En el último tiempo se están investigando otros estrógenos para disminuir el riesgo de complicaciones vasculares y vuelve a aparecer la antigua clormadinona. Nos mantenemos a la espera de nuevas progestinas y otros estrógenos naturales.

En este punto hay que hacer referencia al tal vez el más antiguo de los estudios clínicos y que se ha mantenido en marcha hasta el día de hoy, que se inició en mayo de 1968 y que convocó a 1400 médicos generales del Reino Unido y que inscribieron aproximadamente 23 mil mujeres usuarias de anticonceptivos orales y un número similar de mujeres que nunca habían usado este método anticonceptivo. Analizando esta cohorte, Hannaford y cols (7) publicaron recientemente en el British Medical Journal un análisis estadístico sobre 46.112 de estas mujeres observadas por hasta 39 años, sumando 378.006 mujeres año de observación entre las que nunca usaron anticonceptivos orales y 819.175 entre las que siempre lo habían utilizado. Este estudio agrega al conocimiento que se tenía el hecho que la utilización de la anticoncepción oral no se asoció a un aumento del riesgo de muerte a largo plazo, lo que nos permite seguir ayudando a la mujer que busca métodos anticonceptivos de gran eficacia y que le permitan en forma inocua utilizarlos por períodos prolongados de la vida.

Hasta aquí, la historia ha sido clara y sigue siendo diáfana, con una aceptación generalizada por el nuevo sistema anticonceptivo. Pero ahora debemos preguntarnos el por qué de esa difundida aceptación, y la respuesta es que el mundo femenino estaba esperando, y sigue necesitando, sistemas o métodos que aliviaran los riesgos y las complicaciones de una maternidad elevada, no deseada y que se solucionaba muy frecuentemente con la interrupción del embarazo en situación de riesgo, lo que provocaba una alta morbilidad y una inaceptable mortalidad.

A fines de los años 50 se contaba solamente con métodos anticonceptivos poco seguros y de difícil manejo, en los que destacaba el diafragma, ya no utilizado por la población femenina chilena. Posteriormente, y desde los trabajos del Dr. Jaime Zipper, a quien se le debe un merecido reconocimiento, cada vez más mujeres estaban utilizando un dispositivo intrauterino de plástico, que posteriormente fue reemplazado por la $\mathrm{T}$ con cobre 380 A de amplia utilización hoy día. Pero era necesario contar con un método anticonceptivo que cumpliera 
con los requisitos de eficacia, inocuidad y de fácil utilización, con pocos efectos secundarios, cuyo uso pudiera ser controlado por la misma mujer.

En esos años, y específicamente en 1964, la mortalidad materna en Chile era la más alta de toda América, llegando a 316 muertes por 100.000 nacidos vivos, de los cuales el aborto era responsable de 166 muertes por cien mil nacidos vivos $(8,9)$. Esto significaba que en el curso de un año en Chile morían alrededor de 870 mujeres por embarazo, parto y puerperio, y de ellas, 360 mujeres morían debido a las complicaciones del aborto realizado en condiciones de riesgo como producto del embarazo no deseado. Esto equivale a que se moría en promedio juna mujer cada día!, sin poder cuantificar los daños físicos y sicológicos de aquellas que lograban sobrevivir.

Esta situación dramática fue variando paulatinamente, y en directa relación inversa con el aumento de la cobertura anticonceptiva, para llegar a 18,3 muertes por 100.000 nacidos vivos en 2007 , esto último correspondiendo a 44 muertes de las cuales solo 2 son catalogadas como consecuencia de aborto (10), lo que no quiere decir que no existan abortos inducidos en el país. Solo podemos conjeturar su cuantía, ya que desgraciadamente, en su momento oportuno, el gobierno de turno no nos permitió acceder a participar en la Encuesta Mundial de Fecundidad, que tanta y valiosa información nos podría estar entregando.

Como consecuencia de una búsqueda que ha sido característica de todos aquellos países que empiezan a caminar en el desarrollo social, económico y cultural, en esos momentos ya se empezaba a producir una modificación del comportamiento demográfico de la población chilena, producto de la utilización de los métodos referidos. Pero fue la introducción de la píldora en el Servicio de Salud y en el mercado nacional, y la posibilidad de adquirirla sin ser necesario contar con una receta médica, lo que permitió que desde ese momento empezara a aumentar rápidamente la cobertura anticonceptiva, siempre en base a la utilización voluntaria de métodos modernos en las mujeres de edad fértil. Hoy día se necesita receta médica solo para la adquisición de Anticoncepción Hormonal de Emergencia, situación inequitativa que solo logra impedir que las poblaciones con menos recursos tengan alcance a un método ético, eficaz y seguro.

Al igual que en la mayoría de los países de América Latina, a partir de la segunda mitad de la década de 1960, comenzó a disminuir en forma voluntaria el número medio de hijos por mujer, desde 5,4 en el quinquenio 1960-1965 a 2,3 hijos por mujer en el quinquenio 1995-2000, llegando en la actualidad a cifras ligeramente inferiores a 2, nivel levemente menor a la fecundidad registrada en el mundo en el período 1995-2000 (2,3 hijos por mujer). $Y$ para que no nos dejemos impresionar por estas cifras, solo debemos reconocer que si nos remontamos en el tiempo, el nivel de fecundidad de Latinoamérica es hoy similar al que tuvo Europa en 1950. El problema es que nuevamente tenemos que denunciar la mantención de una inequidad reproductiva dada por una fecundidad caracterizada por un hijo más para la población perteneciente al programa Chile Solidario que para el resto.

A pesar de todos los beneficios reconocidos internacionalmente, persisten grupos detractores de la píldora y de los anticonceptivos en general, quienes aducen que de mantener su actual nivel de utilización nos vamos a transformar, a muy corto plazo, en un país envejecido, olvidando que actualmente contamos con la mayor población joven que haya tenido el país en toda su historia, población que tiene un potencial reproductivo que no ha comenzado a ejercer aún, y que ya han iniciado o están por iniciar su vida sexual y necesitarán por lo tanto protegerse con anticonceptivos para no mantener el alto nivel de fecundidad que caracteriza a este grupo, evitando así el drama que significa un embarazo no deseado con todas sus consecuencias ya conocidas, tanto para la madre como para sus hijos. Solo para ejemplificar el hecho, en el año 1975 la población joven era de 1.163.705 en el país, y de acuerdo a proyección de CEPAL, para el año 2050 se espera una cifra muy similar, 1.153.889, con una tasa de fecundidad mantenida en el curso del tiempo de 1,85 hijos por mujer (11).

La historia sanitaria de nuestro país nos revela que la eficiencia del programa de planificación familiar y las coberturas de uso de anticonceptivos conseguidas, fueron posibles desde su origen gracias a la estructura de nuestro sistema de salud (12), que mantiene una cobertura sobre todo el territorio nacional, a la presencia de los recién creados Médicos Generales de Zona a fines de los años 50, a la capacitación y profesionalismo de las matronas y a la temprana y permanente presencia de APROFA. Esto último permitió contar en forma permanente y sin costo para el país, de un suministro continuo y adecuado a las necesidades anticonceptivas de la población, hasta comienzos de los años 90 , en que, dado el nivel de desarrollo existente Chile, éste deja de ser país prioritario para los donantes internacionales y el Estado debe hacerse cargo de obtener los suministros necesarios para garantizar sin discriminación alguna, la disponibilidad, la accesibilidad física y segura, la información adecuada para conseguir la aceptabilidad del uso de los an- 
ticonceptivos, manteniendo una calidad adecuada de los establecimientos con una capacitación permanente del personal de los servicios públicos de salud y centros de atención de la salud.

Hay que reconocer sí, que a pesar de no contar con programas adecuados de educación sexual en la instrucción formal, las mujeres chilenas, salvo el grupo adolescente, en estos 50 años han logrado mejorar sus expectativas con respecto a ejercer el derecho a decidir libre y responsablemente el número de hijos y el intervalo entre ellos, a obtener información y acceder a métodos seguros, eficaces y aceptables para la regulación voluntaria de la fecundidad, recibiendo en general servicios adecuados de atención de la salud que permitan embarazos y partos sin riesgos y alcanzando un razonable nivel de salud sexual y reproductiva, pudiendo adoptar decisiones en materia sexual sin ningún tipo de discriminación, coacción o violencia.

Dr. Guillermo Galán Ch. Past President APROFA.

\section{REFERENCIAS}

1. Marker RE, Lopez J. Steroidal sapogenins; the position of the double bond in yuccagenin and kammogenin. J Am Chem Soc 1947;69(10):2401.

2. Marker RE, Lopez J. Steroidal sapogenins; biogenesis of the steroidal sapogenins in Agaves, Manfreda and Hesperaloe. J Am Chem Soc 1947;69(10):2403.

3. Pincus G, Rock J, Garcia CR, et al. Fertility con- trol with oral medication. Am J Obstet Gynecol 1958;75(6):1333-46.

4. Pincus G, Rock J, Chang MC, Garcia CR. Effects of certain 19-nor steroids on reproductive processes and fertility. Fed Proc 1959;18:1051-6.

5. Pincus $\mathrm{G}$. Control of conception by hormonal steroids. Science 1966 29;153(735):493-500.

6. Dionne P, Vickerson F. A double-blind comparison of two oral contraceptives containing $50 \mathrm{mu}$ g. and $30 \mathrm{mu}$ g. ethinyl estradiol. Curr Ther Res Clin Exp 1974;16(4):281-8.

7. Hannaford PC, Iversen L, Macfarlane TV, Elliott AM, Angus V, Lee AJ. Mortality among contraceptive pill users: cohort evidence from Royal College of General Practitioners' Oral Contraception Study. BMJ 2010;340:c927. doi: 10.1136/bmj.c927.

8. Armijo R, Monreal T. [The problem of induced abortion in Chile]. Bol Oficina Sanit Panam 1966; 60(1): 39-45. [Article in Spanish].

9. Viel B, Campos W. Chilean history of infant maternal mortality, 1940-1985. Perspec Int Planif Fam 1987;(Spec №): 24-8.

10. Donoso E. Tras el cumplimiento del $5^{\circ}$ objetivo del milenio: mortalidad materna, Chile 2007. Rev Chil Obstet Ginecol 2010;75(1):1-2.

11. CEPAL/ECLAC. Naciones Unidas. América Latina: Fecundidad, 1950-2050. Boletín Demográfico. № 68. 2001. Disponible en: http://www.eclac.org/publicaciones/xml/3/7463/LCG2136_inicio.pdf

12. Gobierno de Chile. Ministerio de Salud. Normas Nacionales sobre Regulación de la Fertilidad. Disponible en: http://www.redsalud.gov.cl/portal/url/item/795c63c aff4ede9fe04001011f014bf2.pdf 